OPEN ACCESS

Edited by:

Sophia George,

University of Miami, United States

Reviewed by:

Sikandar Shaikh

Shadan Hospital and Institute of

Medical Sciences, India

Nguyen Minh Duc,

Pham Ngoc Thach University of

Medicine, Vietnam

*Correspondence:

Jiong $\mathrm{Cal}$

jiong_cai@163.com

Pan Wang

1298178828@qq.com

${ }^{\dagger}$ These authors have contributed equally to this work

Specialty section:

This article was submitted to

Gynecological Oncology,

a section of the journal

Frontiers in Oncology

Received: 21 December 2021

Accepted: 17 January 2022

Published: 09 February 2022

Citation:

Hu X, Liang Z, Zhang C, Wang G,

Cai J and Wang $P$ (2022)

The Diagnostic Performance of Maximum Uptake Value and Apparent

Diffusion Coefficient in Differentiating

Benign and Malignant Ovarian or Adnexal Masses: A Meta-Analysis.

Front. Oncol. 12:840433.

doi: 10.3389/fonc.2022.840433

\section{The Diagnostic Performance of Maximum Uptake Value and Apparent Diffusion Coefficient in Differentiating Benign and Malignant Ovarian or Adnexal Masses: A Meta-Analysis}

\author{
Xianwen $\mathrm{Hu}^{1 \dagger}$, Zhigang Liang ${ }^{1 \dagger}$, Chuanqin Zhang ${ }^{1}$, Guanlian Wang ${ }^{2}$, Jiong Cai ${ }^{1 *}$ \\ and Pan Wang ${ }^{1 *}$
}

${ }^{1}$ Department of Nuclear Medicine, Affiliated Hospital of Zunyi Medical University, Zunyi, China, ${ }^{2}$ Research and Development Department, Jiangsu Yuanben Biotechnology Co., Ltd., Zunyi, China

Objective: The purpose of this meta-analysis was to provide evidence for using maximum uptake value (SUVmax) and apparent diffusion coefficient (ADC) to quantitatively differentiate benign and malignant ovarian or adnexal masses, and to indirectly compare their diagnostic performance.

Material and Methods: The association between SUVmax, ADC and ovarian or adnexal benign and malignant masses was searched in PubMed, Cochrane Library, and Embase databases until October 1, 2021. Two authors independently extracted the data. Studies included in the analysis were required to provide data for the construction of a $2 \times 2$ contingency table to evaluate the diagnostic performance of SUVmax or ADC in differentiating benign and malignant ovarian or adnexal masses. The quality of the enrolled studies was evaluated by Quality Assessment of Diagnostic Accuracy Studies-2 (QUADAS-2) instrument, and the meta-analysis was conducted using Stata software version 14.0. Forest plots were generated according to the sensitivity and specificity of SUVmax and ADC, and metaregression analysis was further used to assess heterogeneity between studies.

Results: A total of 14 studies were finally included in this meta-analysis by gradually excluding duplicate literatures, conference abstracts, guidelines, reviews, case reports, animal studies and so on. The pooled sensitivity and specificity of SUVmax for quantitative differentiation of benign and malignant ovarian or adnexal masses were 0.88 and 0.89 , respectively, and the pooled sensitivity and specificity for ADC were 0.87 and 0.80 , respectively.

Conclusion: Quantitative SUVmax and ADC values have good diagnostic performance in differentiating benign and malignant ovarian or adnexal masses, and SUVmax has higher accuracy than ADC. Future prospective studies with large sample sizes are needed for the analysis of the role of SUVmax and ADC in the differentiation of benign and malignant ovarian or adnexal masses.

Keywords: ovarian cancer, maximum uptake value, apparent diffusion coefficient, PET/CT, diffusion weighted imaging 


\section{INTRODUCTION}

Ovarian cancer is one of the most common and deadly malignant tumors of the female reproductive tract. According to the latest statistics, 314,000 new ovarian cancer patients and 207,000 new deaths were due to ovarian cancer in the world in 2020, seriously threatening the lives and health of women (1). Ovarian neoplasms have a variety of morphological characteristics and genetic and epigenetic changes, and lack of specific clinical manifestations, so it is challenging to distinguish benign from malignant ovarian lesions (2). Approximately $60 \%$ of ovarian cancer patients are already at an advanced stage when diagnosed, resulting in a five-year survival rate of only about $27 \%$ for these patients $(3,4)$. Therefore, it is of great significance to find a highly sensitive and specific diagnostic method for correct diagnosis, clinical management, and prognosis evaluation of ovarian cancer.

Imaging examination and serum tumor marker examination are common screening methods for gynecological diseases. However, the increase of serum tumor markers is not specific for the diagnosis of ovarian cancer, and can also be positive in some benign diseases such as pelvic inflammatory disease, ovarian cyst, and endometriosis (5). Ultrasound is the most commonly used imaging method for ovarian or adnexal lesions, but it has some limitations due to low resolution, obesity, and interference of intestinal gas artifacts (6). Computed tomography (CT) and magnetic resonance imaging (MRI) can provide anatomic information of ovarian lesions and surrounding tissues, which is of great clinical significance to determine the nature, extent of involvement, and treatment decision of ovarian lesions. Compared with CT, MRI has a higher soft tissue resolution. As a sequence of MRI, diffusion weighted imaging (DWI) has been proved to be of high diagnostic value in differentiating benign and malignant adnexal masses, especially entirely solid non-fatty, nonhemorrhagic masses, or complex masses that are either septated cysts or combined solid and cystic masses (7). However, DWI still presents a great challenge in differentiating mature teratoma from benign tumor and ovarian fibroma from malignant tumor (8). Positron emission tomography (PET)/CT imaging integrates $\mathrm{CT}$ and PET into one machine, simultaneously realizing the combination of anatomical imaging and functional imaging, which can intuitively reflect the changes of tumor cell metabolism and make accurate diagnosis in the early stage of tumor. Previous meta-analyses have shown that PET/CT has a high accuracy in differentiating ovarian or adnexal benign and malignant tumors (9). However, the quality of the above study was limited by the different methods used in the included studies and the incomplete use of quantitative data for differentiating ovarian benign and malignant tumors. In order to solve this problem, this study conducted a meta-analysis based on published high-quality studies to quantitatively evaluate the diagnostic performance of maximum uptake value (SUVmax) of ${ }^{18}$ F-FDG PET/CT and apparent diffusion coefficient (ADC) values of DWI-MRI in differentiating benign and malignant ovarian tumors.

\section{MATERIALS AND METHODS}

\section{Study Search Strategy}

The present meta-analysis was performed in accordance with the 2009 PRISMA guidelines (10). The Pubmed, Cochrane Library, and Embase databases were searched for articles reporting on SUVmax or ADC values in the differentiation of ovarian or adnexal masses and included in the studies. The search terms for a complete search strategy were as follows: ("PET/CT" OR "PETCT" OR "positron emission tomography/computed tomography" OR "positron emission tomography-computed tomography" OR "SUVmax" OR "Maximum standard uptake value" OR "MR" OR "Magnetic Resonance" OR "diffusion weighted imaging" OR "diffusion magnetic resonance imaging" OR "apparent diffusion coefficient" OR "ADC") AND ("ovarian neoplasms" OR "ovary neoplasms" OR "ovary cancer" OR "ovarian cancer" OR "cancer of ovary" OR "ovarian tumor" OR "ovarian carcinoma" OR "adnexal mass" OR "adnexal lesions"). Original studies published in English with a deadline of October 1, 2021 were included. Articles were screened by two reviewers with 10 years of meta-analysis writing experience. All articles that might be suitable for this study were reserved after reading abstracts. In case of disputes, the team members discussed them until a consensus was reached.

\section{Study Selection and Exclusion Criteria}

The published studies included in the current meta-analysis are required to meet the following inclusion criteria: i) articles whose full text is in English before the deadline for publication on October 1, 2021; ii) prospective or retrospective study of accuracy in differentiating benign and malignant ovarian or adjunctive masses using SUVmax of ${ }^{18}$ F-FDG PET/CT and/or ADC values of DWI-MRI; iii) studies can directly extract or calculate the true positive, false positive, false negative, and true negative values based on the sensitivity, specificity, negative predictive value, positive predictive value in the article to construct a $2 \times 2$ contingency table; and iv) interpretation of the results should include at least histopathological findings. The exclusion criteria included: i) studies with sample sizes less than 30 patients; ii) although studies used SUVmax or/and ADC values to differentiate benign and malignant ovarian or adnexal masses, the cut-off value was not described in detail; iii) studies are published duplicately or contain overlapping data; iv) for studies of ADC values differentiating benign from malignant ovarian or adjunctive masses, magnetic field intensity of MRI should be described and studies of less than 1.5 Tesla (T) should be excluded; and v) for SUVmax, studies using tracers other than ${ }^{18} \mathrm{~F}$-FDG.

\section{Data Extraction and Quality Assessment}

A standard data extraction form were used from enrolled studies, and descriptive information on a range of factors was independently collected, namely, the first author, publication year, country, study design type (retrospective or prospective), number of patients, mean age, patient selection (consecutive or nonconsecutive), positive reference standard, the cutoff value of 
SUVmax for PET/CT, and ADC value for DWI-MRI, the interval time between index tests and HP, true-positive (TP), false-positive (FP), false-negative (FN), and true-negative (TN) results from the included studies. In addition, data were extracted on the characteristics and techniques of the scanners used in the study, such as magnetic field strength for MRI, CT technology (whether contrast agent contrast enhancement scan is used) for PET/CT and so on. The Quality Assessment of Diagnostic Accuracy Studies-2 (QUADAS-2) was used to evaluate the quality of the enrolled studies (11). Data extraction and critical evaluation of article quality are conducted independently by two authors, and they will negotiate together until a consensus was reached in case of disputes.

\section{Statistical Analyses}

The current meta-analysis was conducted using Stata software (version 14.0; Stata Corporation, College Station, TX, USA). The specificity, sensitivity, positive likelihood ratio (PLR), negative likelihood ratio (NLR), diagnostic odds ratio (DOR) and summary receiver operating characteristic (SROC) curve to count the area under the curve (AUC) with their 95\% confidence intervals (CIs) calculated based on the TP, FP, FN, and $\mathrm{TN}$ values extracted from the enrolled studies were used to evaluate the diagnostic performance of SUVmax and ADC value in differentiating between benign and malignant ovarian or adnexal masses. The general estimates of sensitivity and specificity of enrolled studies were calculated using hierarchical logistic regression models, namely, hierarchical summary receiver operating characteristics (HSROC) models and concomitant variables. The HSROC curve with a 95\% confidence and prediction region is used to plot its sensitivity and specificity results. Heterogeneity between studies was assessed using Cochran's Q test and Higgins $I^{2}$ test (12). For Cochran's Q test, $\mathrm{P}<0.05$ was the test standard, indicating that there was heterogeneity among the enrolled studies. The criteria for evaluating the degree of heterogeneity using Higgins $\mathrm{I}^{2}$ test is: inconsistency index $\left(\mathrm{I}^{2}\right)<50 \%$ was deemed as irrelevant heterogeneity; $\mathrm{I}^{2}=50-80 \%$ was considered to be moderate heterogeneity; $\mathrm{I}^{2}>80 \%$ indicated significant heterogeneity. The funnel plots and Deeks' asymmetry tests were used as the assessment of publication bias for ADC value of DWI-MRI and SUVmax of ${ }^{18}$ F-FDG PET/CT (13). A two-sample Z-test was conducted to evaluate the difference in diagnostic performance between the two diagnostic methods, and $\mathrm{p}<0.05$ was considered to be statistical significance in the diagnostic performance of the two methods in the quantitative differentiation of benign and malignant ovarian or adnexal masses.

\section{RESULTS}

\section{Literature Search}

A total of 6,302 articles were retrieved from three databases by subject terms, consisting of 3,554 articles in PubMed, 2,361 articles in Embase and 387 articles in Cochrane Library. After gradually ruling out overlapping, irrelevant comments, guidelines, conferences, case studies, animal studies, and articles not in the field of interest, etc., 6,174 articles were excluded, and the remaining 128 potentially eligible original texts were further evaluated. By carefully reading the full text, 116 articles were further excluded, including studies that not the full text was published in English, data could not be extracted to construct a contingency table, and papers were in areas of noninterest. Finally, a total of 14 articles, namely, 7 using SUVmax of

${ }^{18}$ F-FDG PET/CT and 7 using ADC values of DWI-MRI to quantitatively differentiate the diagnostic performance of benign and malignant ovarian or adnexal masses were included in this meta-analysis (14-27). The detailed retrieval process of the literature is shown in Figure 1.

\section{Study Characteristics}

The 14 enrolled studies included a total of 1,317 patients with 1,373 masses, consisting of 3 prospective studies, 10 retrospective studies, and 1 was unspecified. The enrolled studies were published from 2007 to 2020, with sample sizes ranging from 30 to 191 and mean ages of patients ranging from 39.3 to 64 . At least histopathological results were used as a reference for positive interpretation in all of the enrolled studies, and followup results were also used in two of the studies. Follow-up included imaging findings and serum tumor markers for at least six months. For SUVmax of ${ }^{18}$ F-FDG PET/CT, two studies used both non-contrast-enhanced scanning and contrast-enhanced CT technique $(16,19)$. As for ADC, five studies used magnetic field strengths of $1.5(\mathrm{~T})$, one of $3.0 \mathrm{~T}$ and one of both. The detailed characteristics of the enrolled studies are summarized in Table 1. The characteristics of the enrolled studies to differentiate benign and malignant ovarian or adnexal masses by quantitative SUVmax of PET/CT are shown in Table S1, and the characteristics for quantitative ADC values of DWI-MRI are shown in Table S2.

\section{Quality Evaluation}

The quality of all 14 enrolled studies met at least five of the 7 reference criteria (namely, four items in the risk of bias, patient selection, index test, reference standard, flow and timing and three in application concerns, patient selection, index test, reference standard) was therefore considered satisfactory. For the risk of reference standard bias, all studies at least used histopathological findings as a positive interpretation and were considered low risk. Regarding the risk of bias for flow and time, 10 of the 14 studies did not report the time interval between the index and the reference standard test, so the risk of bias was unclear. All the patients in the enrolled studies were suspected of having ovarian or adnexal masses by ultrasound or serum tumor markers, so the risk of publication bias and application concerns in patient selection were considered low. The results of the QUADAS-2 assessment are shown in Table 2.

\section{Diagnostic Accuracy}

A total of seven enrolled studies using SUVmax to differentiate ovarian or adnexal benign and malignant tumors had sensitivity ranging from 0.71 (95\% CI, $0.42-0.92)$ to 1.0 (95\% CI, $0.81-1.00)$ and specificity ranging from 0.77 (95\% CI, 0.56-0.91) to $1.0(95 \%$ CI, $0.81-1.00)$, with a pooled sensitivity and specificity of 0.88 


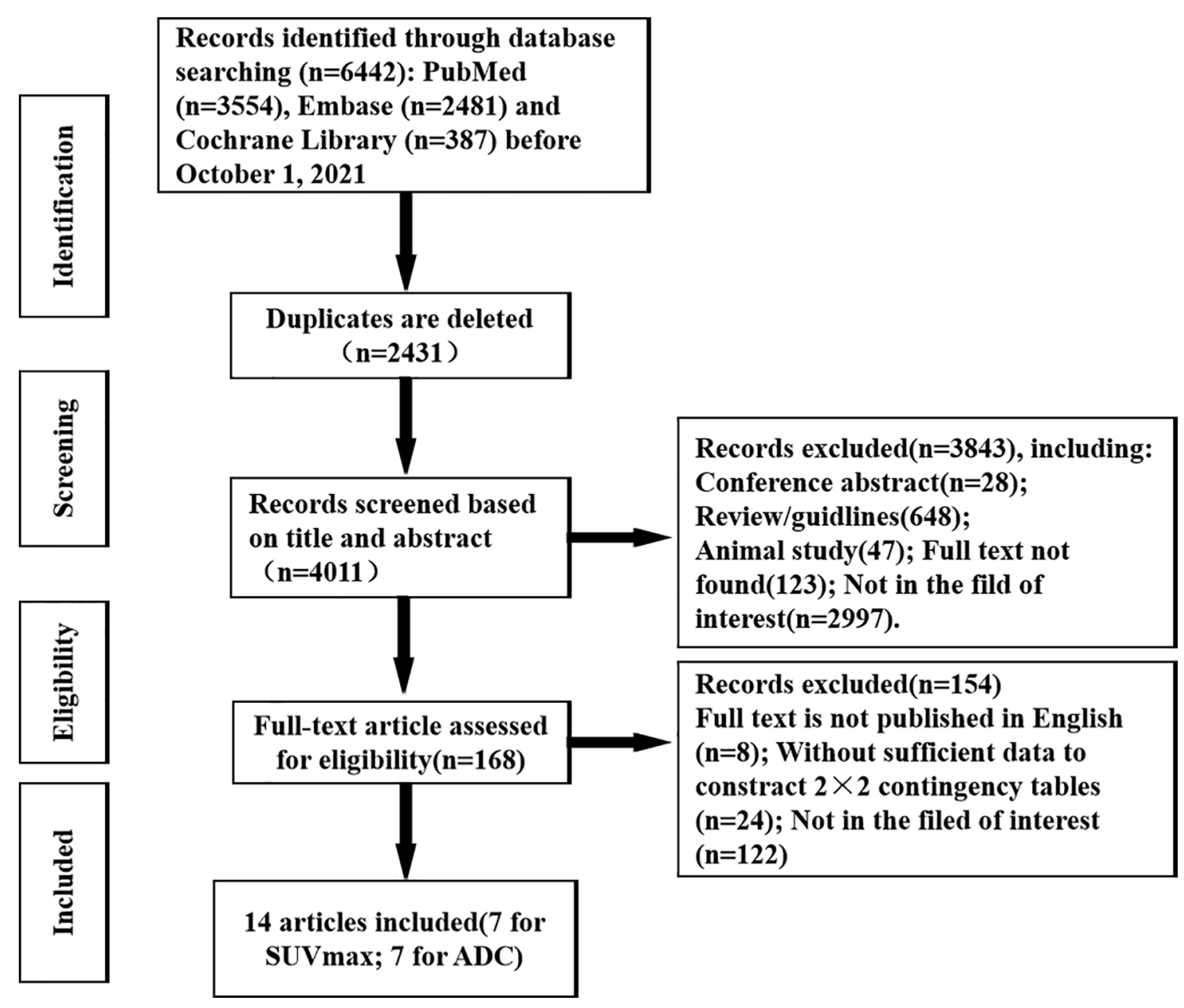

FIGURE 1 | Flow chart of the research selection process.

(95\% CI, 0.81-0.93) and 0.89 (95\% CI, 0.81-0.94), respectively, as shown in Figure 2. Both Cochran's Q test and Higgins $\mathrm{I}^{2}$ test showed moderate heterogeneity in sensitivity $(\mathrm{Q}=15.02, \mathrm{p}=$ $\left.0.02 ; \mathrm{I}^{2}=60.24\right)$ and specificity $\left(\mathrm{Q}=12.95, \mathrm{p}=0.04 ; \mathrm{I}^{2}=53.68\right)$.

Also, a total of 7 studies using ADC values of DWI-MRI evaluated the diagnostic performance of benign and malignant ovarian or adnexal masses, with sensitivity ranging from 0.65 (95\% CI, $0.43-0.84)$ to 0.93 (95\% CI, 0.83-0.98) and specificity ranging from $0.61(95 \% \mathrm{CI}, 0.51-0.70)$ to 0.89 (95\% CI, $0.76-$ $0.96)$, for a pooled sensitivity and specificity of 0.87 (95\% CI, $0.80-0.92)$ and 0.80 (95\% CI, 0.71-0.87), respectively, as shown in Figure 3. Both Cochran's Q test and Higgins $\mathrm{I}^{2}$ test showed heterogeneity among studies in sensitivity $(\mathrm{Q}=23.28, p<0.01$; $\left.\mathrm{I}^{2}=74.23\right)$ and specificity $\left(\mathrm{Q}=22.87, p<0.01 ; \mathrm{I}^{2}=73.76\right)$, too.

The calculated mean SUVmax for benign and malignant ovarian or adnexal masses was 2.155 and 9.588, respectively, with significant statistical difference $(p<0.001)$.The graphical distribution of SUVmax in benign and malignant ovarian or adnexal masses is shown in Figure 4A. The calculated mean $\mathrm{ADC}$ values for benign and malignant ovarian or adnexal masses were $1.364 \times 10^{-3} \mathrm{~mm}^{2} / \mathrm{s}$ and $0.933 \times 10^{-3} \mathrm{~mm}^{2} / \mathrm{s}$, respectively, also showing significant statistical differences (Figure 4B). The PLR of SUVmax of ${ }^{18}$ F-FDG PET/CT and ADC of DWI-MRI were 7.8 (95\% CI, 4.5-13.6) and 4.4 (95\% CI, 2.9-6.8), and the NLR was 0.13 (95\% CI, 0.08-0.21) and 0.16 (95\% CI, 0.11-0.25), respectively. The pooled DOR for benign and malignant ovarian or adnexal masses assessed by SUVmax and ADC was $59(95 \%$ CI, 27-128) and 27 (95\% CI, 14-54), with a pooled AUC of 0.95 (95\% CI, 0.92-0.96) and 0.91 (95\% CI, 0.88-0.93), respectively, as shown in Table 3. Overall, there was no statistical difference in sensitivity and specificity of using SUVmax and ADC values in the assessment of benign and malignant ovarian or adnexal masses, with $p$-values of 0.705 and 0.166 , respectively. The area under the SROC curve of SUVmax and ADC are 0.95 (95\% CI, $0.92-0.96)$ and 0.91 (95\% CI, 0.88-0.93), respectively, as shown in Figure 5.

\section{Publication Bias}

A funnel plot for publication bias for SUVmax and ADC by Deeks et al. is shown in Figure 6. The $p$-values of slope 
TABLE 1 | The main characteristics of the enrolled studies.

\begin{tabular}{|c|c|c|c|c|c|c|c|c|c|c|c|}
\hline $\begin{array}{l}\text { Study/Year/ } \\
\text { Country }\end{array}$ & $\begin{array}{l}\text { No. of } \\
\text { patients }\end{array}$ & $\begin{array}{l}\text { Mean } \\
\text { age }\end{array}$ & $\begin{array}{l}\text { Study } \\
\text { design }\end{array}$ & Consecutive & \multicolumn{2}{|c|}{ Technique } & $\begin{array}{l}\text { Cutoff } \\
\text { value }\end{array}$ & Reference standard & $\begin{array}{l}\text { interval } \\
\text { time }\end{array}$ & \multicolumn{2}{|c|}{ Mean value } \\
\hline SUVmax & & & & & & & & & & benign & malignant \\
\hline $\begin{array}{l}\text { Castellucci/2007/ } \\
\text { Italy (14) }\end{array}$ & 50 & 64 & $P$ & Yes & \multicolumn{2}{|c|}{$\begin{array}{l}\text { PET/CT } \\
\text { (non-CE) }\end{array}$} & 3.0 & $\mathrm{HP}+$ follow-up $>6$ months & $\leq 2 \mathrm{~W}$ & $N R$ & NR \\
\hline $\begin{array}{l}\text { Yamamoto/2008/ } \\
\text { Japan (15) }\end{array}$ & 30 & 47.7 & $P$ & Yes & \multicolumn{2}{|c|}{$\begin{array}{l}\text { PET/CT } \\
\text { (non-CE) }\end{array}$} & 3.0 & $\mathrm{HP}$ & $N R$ & 1.74 & 9.32 \\
\hline $\begin{array}{l}\text { Kitajima/2011/Japan } \\
\text { (16) }\end{array}$ & $\begin{array}{l}108(111 \\
\text { tumors })\end{array}$ & 55.4 & NR & NR & \multicolumn{2}{|c|}{$\begin{array}{l}\text { PET/CT } \\
\text { (non-CE and } \\
\text { CE) }\end{array}$} & 2.55 & $\mathrm{HP}$ & $N R$ & 2.0 & 7.55 \\
\hline $\begin{array}{l}\text { Zytoon/2012/Egypt } \\
\text { (17) }\end{array}$ & 98 & 57.7 & $P$ & yes & \multicolumn{2}{|c|}{$\begin{array}{l}\mathrm{PET} / \mathrm{CT} \\
\text { (non-CE) }\end{array}$} & 4.3 & $\begin{array}{l}\mathrm{HP}+\text { follow-up (imaging + } \\
\text { tumor marker) }\end{array}$ & $\leq 4 \mathrm{~W}$ & NR & NR \\
\hline $\begin{array}{l}\text { Tanizaki/2014/ } \\
\text { Japan (18) }\end{array}$ & 160 & NR & $\mathrm{R}$ & $N R$ & \multicolumn{2}{|c|}{$\begin{array}{l}\text { PET/CT } \\
\text { (non-CE) }\end{array}$} & 2.9 & $\mathrm{HP}$ & NR & NR & NR \\
\hline Lee/2015/Korea (19) & 39 & 51 & $\mathrm{R}$ & NR & \multicolumn{2}{|c|}{$\begin{array}{l}\mathrm{PET} / \mathrm{CT} \\
\text { (non-CE and } \\
\mathrm{CE} \text { ) }\end{array}$} & 2.5 & $\mathrm{HP}$ & $\leq 7 \mathrm{~W}$ & 2.4 & 10.5 \\
\hline $\begin{array}{l}\text { Takagi/2018/Japan } \\
\text { (20) }\end{array}$ & 76 & 59 & $\mathrm{R}$ & NR & \multicolumn{2}{|c|}{$\begin{array}{l}\text { PET/CT } \\
\text { (non-CE) }\end{array}$} & 3.97 & $\mathrm{HP}$ & NR & 2.48 & 10.98 \\
\hline ADC & & & & & \multirow{2}{*}{\multicolumn{2}{|c|}{$\begin{array}{l}b \\
\text { value } \\
0,1000\end{array}$}} & $\begin{array}{l}\left(10^{-3} \mathrm{~s} /\right. \\
\left.\mathrm{mm}^{2}\right)\end{array}$ & & & \multicolumn{2}{|c|}{$10^{-3} \mathrm{~s} / \mathrm{mm}^{2}$} \\
\hline Li/2012/China (21) & $\begin{array}{l}127(131 \\
\text { tumors) }\end{array}$ & 59.9 & $\mathrm{R}$ & Yes & & & 1.25 & $\mathrm{HP}$ & NR & 1.69 & 1.03 \\
\hline $\begin{array}{l}\text { Zhang/2012/China } \\
\text { (22) }\end{array}$ & $\begin{array}{l}191(202 \\
\text { tumors) }\end{array}$ & 56.5 & $\mathrm{R}$ & Yes & $\begin{array}{l}\mathrm{MRI} \\
(1.5 \mathrm{~T})\end{array}$ & 0,1000 & 1.2 & $\mathrm{HP}$ & NR & 1.22 & 0.91 \\
\hline Fan/2015/China (23) & 64 & 46.7 & $R$ & NR & $\begin{array}{l}\mathrm{MRI} \\
(3.0 \mathrm{~T})\end{array}$ & 0,1000 & 0.878 & $\mathrm{HP}$ & NR & 1.325 & 0.878 \\
\hline $\begin{array}{l}\text { Zhang/2019/China } \\
\text { (24) }\end{array}$ & 85 & 52.7 & $\mathrm{R}$ & Yes & $\begin{array}{l}\mathrm{MRI} \\
(1.5 \mathrm{~T})\end{array}$ & 0,800 & 1.162 & $\mathrm{HP}$ & $N R$ & NR & $N R$ \\
\hline $\begin{array}{l}\text { Türkoglu/2020/ } \\
\text { Turkey (25) }\end{array}$ & 43 & 51.26 & $\mathrm{R}$ & Yes & $\begin{array}{l}\mathrm{MRI} \\
(1.5 \mathrm{~T})\end{array}$ & 0,800 & 0.93 & $\mathrm{HP}$ & $\leq 1 \mathrm{~W}$ & 1.37 & 0.92 \\
\hline $\begin{array}{l}\text { Mansour/2015/ } \\
\text { Egypt (26) }\end{array}$ & $\begin{array}{c}197 \\
\text { (235tumors) }\end{array}$ & 39.3 & $\mathrm{R}$ & Yes & $\begin{array}{l}\mathrm{MRI} \\
(1.5 \mathrm{~T})\end{array}$ & 0,1000 & 1.2 & $\mathrm{HP}$ & NR & 1.2 & 0.83 \\
\hline $\begin{array}{l}\text { Takeuchi/2010/ } \\
\text { Japan (27) }\end{array}$ & 49 & 59 & $R$ & Yes & $\begin{array}{l}\mathrm{MRI} \\
(1.5 / \\
3.0 T)\end{array}$ & 0,800 & 1.15 & $\mathrm{HP}$ & NR & 1.38 & 1.03 \\
\hline
\end{tabular}

HP, Histopathology; P, Prospective; R, Retrospective; CE, Contrast enhancement; non-CE, none contrast enhancement; NR, Not report; W, Week; MRI, Magnetic resonance imaging; PET/CT, Positron emission computer/Computed tomography; T, Tesla; SUVmax, Maximum uptake value; ADC, Apparent diffusion coefficient.

TABLE 2 | Risk of bias and application concerns for included studies were assessed by the QUQUADAS-2 tool.

\begin{tabular}{|c|c|c|c|c|c|c|c|}
\hline \multirow[t]{2}{*}{ Study } & \multicolumn{4}{|c|}{ Risk of bias } & \multicolumn{3}{|c|}{ Application concerns } \\
\hline & Patient selection & Index test & Reference standard & Flow and timing & Patient selection & Index test & Reference standard \\
\hline Castellucci/2007 (14) & $\mathrm{L}$ & $U$ & $\mathrm{~L}$ & $\mathrm{~L}$ & $\mathrm{~L}$ & $\mathrm{~L}$ & $\mathrm{~L}$ \\
\hline Yamamoto/2008 (15) & $\mathrm{L}$ & $\mathrm{L}$ & $\mathrm{L}$ & $U$ & $\mathrm{~L}$ & $\mathrm{~L}$ & $\mathrm{~L}$ \\
\hline Kitajima/2011 (16) & $\mathrm{L}$ & $\mathrm{L}$ & $\mathrm{L}$ & $U$ & $\mathrm{~L}$ & $\mathrm{~L}$ & $\mathrm{~L}$ \\
\hline Zytoon/2012 (17) & $\mathrm{L}$ & $\mathrm{L}$ & $\mathrm{L}$ & $\mathrm{L}$ & $\mathrm{L}$ & $\mathrm{L}$ & $\mathrm{L}$ \\
\hline Tanizaki/2014 (18) & $\mathrm{L}$ & $\mathrm{L}$ & $\mathrm{L}$ & $U$ & $\mathrm{~L}$ & $\mathrm{~L}$ & $\mathrm{~L}$ \\
\hline Lee/2015 (19) & $\mathrm{L}$ & $U$ & $\mathrm{~L}$ & $\mathrm{~L}$ & $\mathrm{~L}$ & $\mathrm{~L}$ & $\mathrm{~L}$ \\
\hline Takagi/2018 (20) & $\mathrm{L}$ & $\mathrm{L}$ & $\mathrm{L}$ & $U$ & $\mathrm{~L}$ & $U$ & $\mathrm{~L}$ \\
\hline $\mathrm{Li} / 2011$ (21) & $\mathrm{L}$ & $\mathrm{L}$ & $\mathrm{L}$ & $U$ & $\mathrm{~L}$ & $\mathrm{~L}$ & $\mathrm{~L}$ \\
\hline Zhang/2012 (22) & $\mathrm{L}$ & $\mathrm{L}$ & $\mathrm{L}$ & $U$ & $\mathrm{~L}$ & $\mathrm{~L}$ & $\mathrm{~L}$ \\
\hline Fan/2015 (23) & $\mathrm{L}$ & $\mathrm{L}$ & $\mathrm{L}$ & $U$ & $\mathrm{~L}$ & $\mathrm{~L}$ & $\mathrm{~L}$ \\
\hline Zhang/2019 (24) & $\mathrm{L}$ & $\mathrm{L}$ & $\mathrm{L}$ & $U$ & $\mathrm{~L}$ & $\mathrm{~L}$ & $\mathrm{~L}$ \\
\hline Türkoğlu/2020 (25) & $L$ & $U$ & $\mathrm{~L}$ & $\mathrm{~L}$ & $\mathrm{~L}$ & $\mathrm{~L}$ & $L$ \\
\hline Mansour/2015 (26) & $\mathrm{L}$ & $\mathrm{L}$ & $\mathrm{L}$ & $U$ & $\mathrm{~L}$ & $U$ & $\mathrm{~L}$ \\
\hline Takeuchi/2010 (27) & $\mathrm{L}$ & $\mathrm{L}$ & $\mathrm{L}$ & $U$ & $\mathrm{~L}$ & $\mathrm{~L}$ & $\mathrm{~L}$ \\
\hline
\end{tabular}

L, low; U, unclear. 


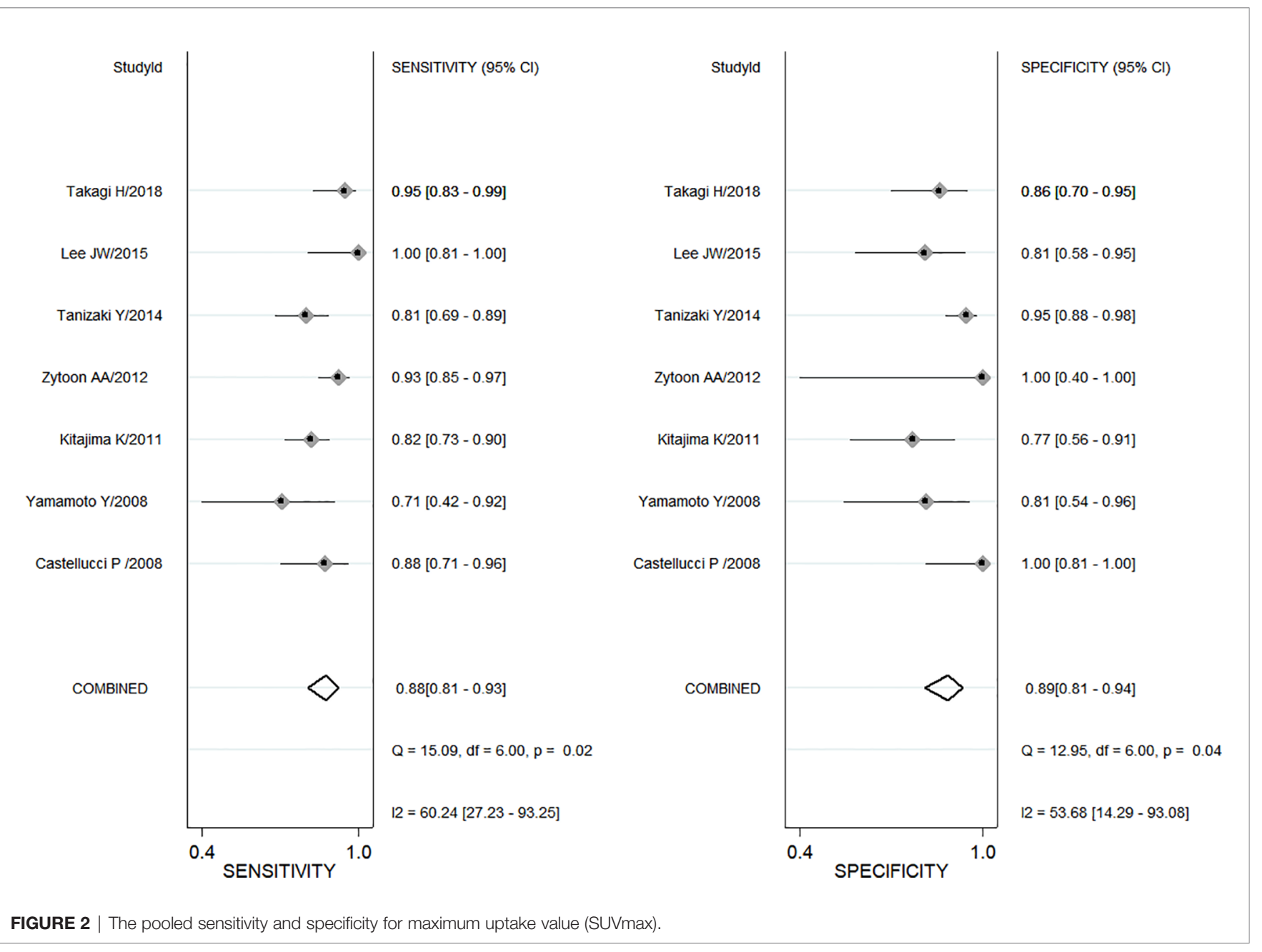

coefficients were 0.97 and 0.44 , respectively, which were both greater than 0.05 , indicating a low possibility of publication bias.

\section{Exploration of Heterogeneity}

To determine the source of heterogeneity between studies, univariate meta-regression analyses of studies using SUVmax and $\mathrm{ADC}$ to differentiate benign and malignant ovarian or adnexal masses were performed, and the results are summarized in Table 4. For the studies of SUVmax, the sensitivity of the cutoff value of SUVmax $\geq 3.0 \quad(0.91 ; 95 \%$ CI: $0.86-0.95)$ is higher than that of SUVmax $<3.0(0.84 ; 95 \% \mathrm{CI}$ : $0.78-0.90)$, with a $p$-value of 0.01 , which may be a factor affecting heterogeneity. In terms of specificity, the sample size of the enrolled study showed heterogeneity $(p=0.03<0.05)$. Specifically, the specificity of a sample size of more than 50 patients is higher than that of a study of 50 or less, being 0.93 (95\% CI: 0.81-0.97) and 0.85 (95\% CI: 0.78-0.99), respectively. The type of study design (prospective or retrospective), the vendor of PET/CT, interval time between FDG administration and scanning, FDG dose and the mean age of the enrolled patients were not factors influencing the heterogeneity between studies (all $p$-values were greater than 0.05 ). As for ADC, the sensitivity of the number of masses enrolled studies greater than 100 was higher than that of a study of 100 or less than, which were 0.91 (95\% CI: $0.88-0.95$ ) and 0.82 (95\% CI: 0.75-0.88), respectively. Moreover, the sensitivity of the study with $6 \mathrm{~mm}$ (0.84; 95\% CI: $0.78-0.95)$ as the scanning slice thickness is lower than that of the study with the scanning slice thickness of $5 \mathrm{~mm}$ (0.89; 95\% CI: 0.80-0.95). Studies performed with a maximal $b$ value of $1,000 \mathrm{~s} / \mathrm{mm}^{2}$ showed lower pooled specificity $(0.79 ; 95 \%$ CI: $0.70-0.89)$ compared with $800 \mathrm{~s} / \mathrm{mm}^{2}$ (0.84; 95\% CI: $0.68-$ $0.95)$. Yet the vendor of MRI, the number of imaging planes, etc. are not factors that affect the heterogeneity between studies $(p>0.05)$.

\section{DISCUSSION}

Although there are many published studies on MRI and ${ }^{18} \mathrm{~F}-\mathrm{FDG}$ $\mathrm{PET} / \mathrm{CT}$ in differentiating benign and malignant ovarian or adjunct masses, the inconsistent positive reference criteria used in these studies lead to a large difference between the results. In order to minimize differences in the range of diagnostic parameters, the current meta-analysis included studies using 

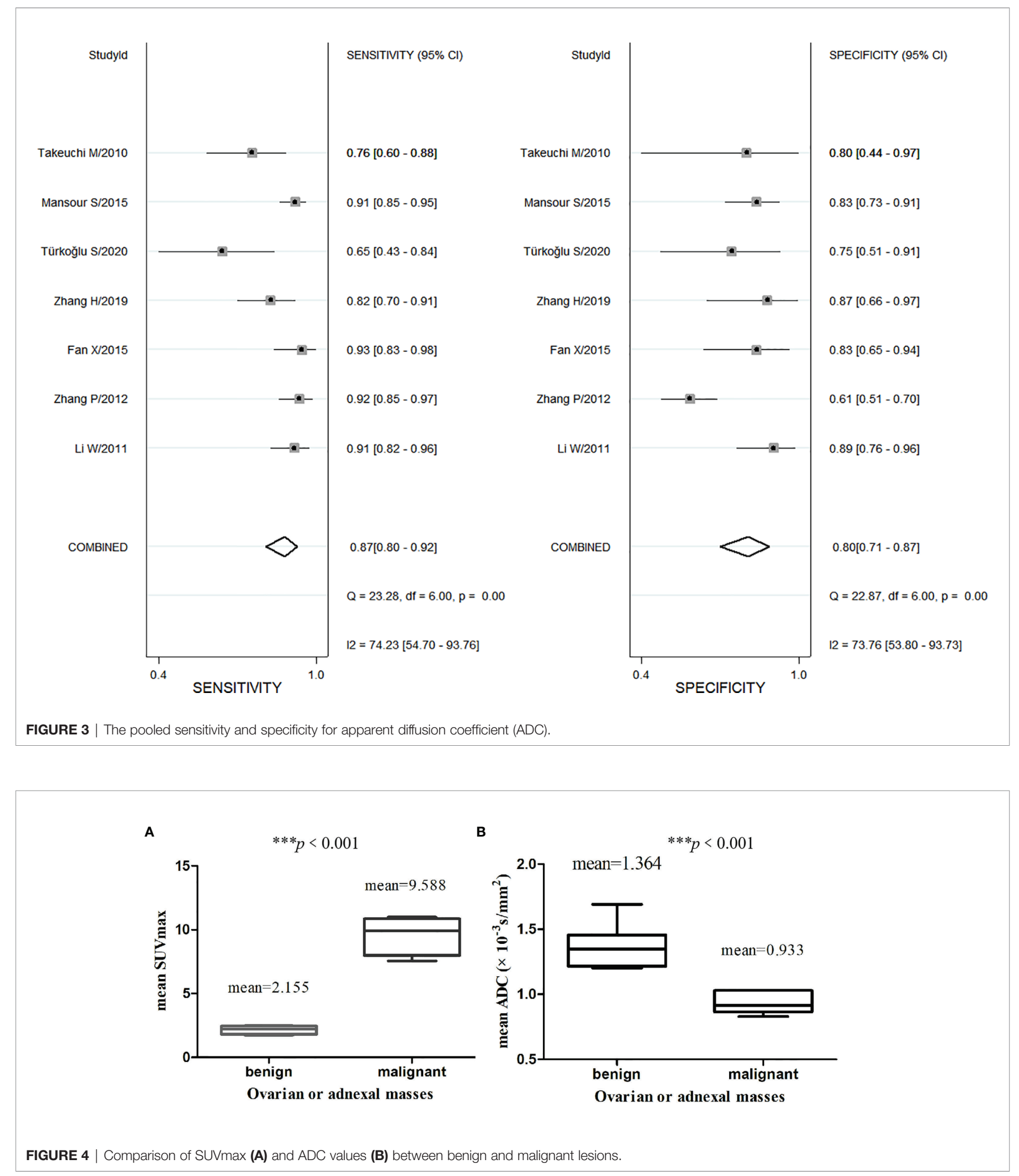

SUVmax and ADC values to differentiate benign and malignant ovarian or adnexal masses to quantitatively evaluate their diagnostic performance and to make indirect comparisons. This comprehensive quantitative meta-analysis covered 1,317 patients with 1,373 masses from 14 studies with a wide range of features. It should be noted that in order to try to maintain consistency of patients enrolled, a study included a large number of patients with a history of primary tumors, so we excluded the 
TABLE 3 | Summary of the diagnostic performance characteristics of SUVmax and ADC value in differentiating benign and malignant ovarian or adnexal masses.

\begin{tabular}{|c|c|c|c|c|}
\hline \multirow[t]{2}{*}{ Parameter } & \multicolumn{2}{|c|}{ SUVmax } & \multicolumn{2}{|c|}{ ADC value } \\
\hline & Estimate & $95 \% \mathrm{Cl}$ & Estimate & $95 \% \mathrm{Cl}$ \\
\hline Sensitivity & 0.88 & $0.81-0.93$ & 0.87 & $0.80-0.92$ \\
\hline Specificity & 0.89 & $0.81-0.94$ & 0.80 & $0.71-0.87$ \\
\hline PLR & 7.8 & $4.5-13.6$ & 4.4 & 2.9-6.8 \\
\hline NLR & 0.13 & $0.08-0.21$ & 0.16 & $0.11-0.25$ \\
\hline DOR & 59 & $27-128$ & 27 & $14-54$ \\
\hline AUC & 0.95 & $0.92-0.96$ & 0.91 & $0.88-0.93$ \\
\hline
\end{tabular}

PLR, positive likelihood ratio; NLR, Negative likelihood ratio; DOR, Diagnostic Odds Ratio; AUC, Area under curve; SUVmax, Maximum uptake value; ADC, Apparent diffusion coefficient.

A

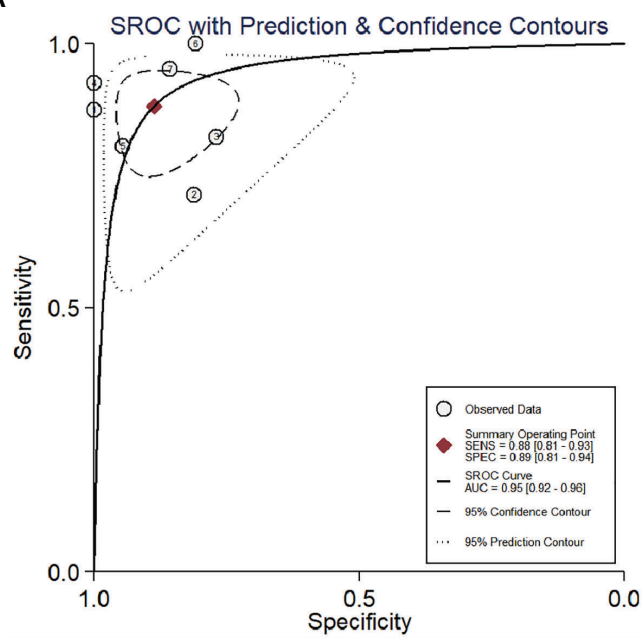

B

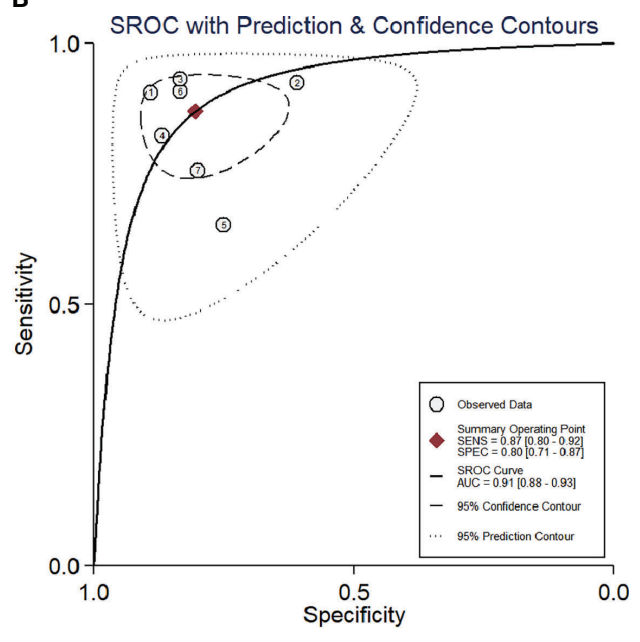

FIGURE 5 | SROC curve of the diagnostic performance of SUVmax (A) and ADC (B) for ovarian cancer. AUC, area under the curve; SENS, sensitivity; SPEC, specificity; SROC, summary receiver operating characteristic.

data of patients with ovarian metastasis from the meta-analysis (19). The results of this meta-analysis showed that SUVmax and $\mathrm{ADC}$ values have good diagnostic performance in quantitatively differentiating benign and malignant ovarian or adnexal masses. Furthermore, the AUC in SUVmax is slightly higher than that in ADC value, being 0.95 and 0.91 respectively.

SUVmax represents the maximum standard uptake value in PET/CT scanning, which is equal to the ratio of imaging agent activity per unit volume of lesion tissue to injection dose, and is usually used as a quantitative indicator of ${ }^{18} \mathrm{~F}$-FDG tracer uptake in tumor tissue. Clinically, SUVmax is usually used to identify benign and malignant tumors and indicate the degree of malignancy of tumors. A number of previous meta-analysis results showed that SUVmax is of great value in the staging, prognosis evaluation and monitoring of treatment response of various malignant tumors, such as breast cancer, cervical cancer and lung cancer (28-33). Moreover, a few meta-analyses results showed that SUVmax was correlated with Ki-67 index $(34,35)$. Our meta-analysis included 7 studies with 561 patients using SUVmax to quantitatively differentiate benign and malignant ovarian or adnexal tumors, and the results also showed good diagnostic accuracy.
ADC is used as a parameter to describe the diffusion speed and range of different water molecules in DWI-MRI, which was first applied to the central nervous system and has been recognized as an indispensable technology in many radiology fields presently (36). Compared with normal tissue or benign lesions, malignant neoplasms are usually composed of multicellular tissue and have limited diffusion in areas of high cell density, thus presenting with reduced diffusion of water molecules and a reduced apparent diffusion coefficient (37). ADC has been proven to have good diagnostic performance in the identification of benign and malignant lesions such as the brain, thyroid, pancreas, and uterus (3842). In addition, ADC has also been confirmed in other studies to better predict the response evaluation of liver cancer, nasopharyngeal cancer, colon cancer with liver metastasis after treatment (43-45). Whether the ADC value can better distinguish benign and malignant ovarian or appendages is different in some previous studies $(46,47)$. However, the results of our study based on a total of 799 masses in 756 patients showed that the sensitivity and specificity of ADC values in the differentiation of ovarian or adnexal benign and malignant masses were 0.87 and 0.80 , respectively, with an AUC of 0.91 , 


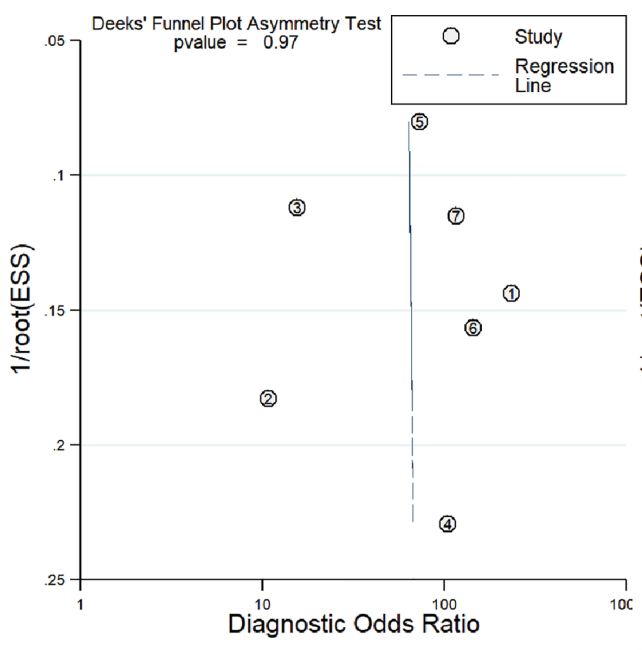

B

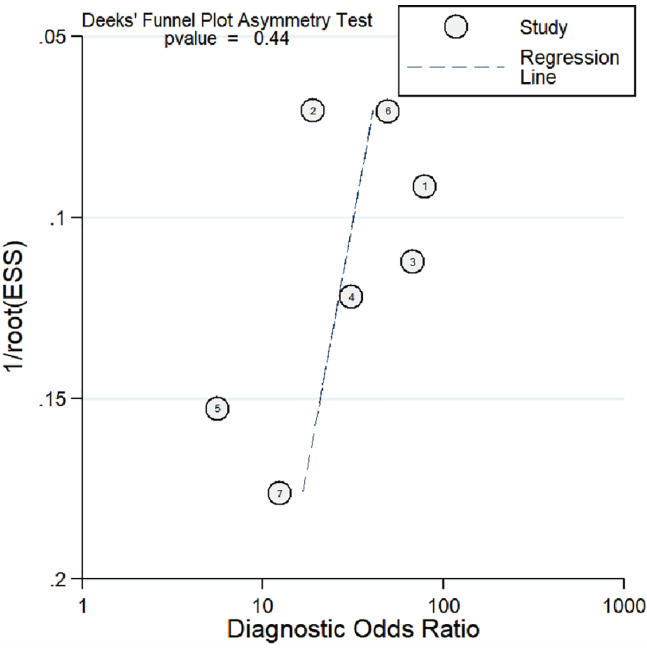

FIGURE 6 | Deeks et al.'s funnel plot for publication bias for SUVmax (A) and ADC (B).

TABLE 4 | The results of meta-regression analysis of SUVmax and ADC to differentiate benign and malignant ovarian or adnexal tumors.

\begin{tabular}{|c|c|c|c|c|c|c|}
\hline Parameter & Category & No. of studies & Sensitivity & $p$-value & Specificity & $p$-value \\
\hline \multicolumn{7}{|l|}{ SUVmax } \\
\hline \multirow[t]{2}{*}{ Design } & Prospective & 3 & 0.88 [0.78-0.98] & 0.19 & $0.92[0.82-1.00]$ & 0.67 \\
\hline & Retrospective & 3 & $0.92[0.84-1.00]$ & & $0.89[0.82-0.96]$ & \\
\hline \multirow[t]{2}{*}{ Mean age } & $\geq 56$ & 3 & $0.92[0.88-0.96]$ & 0.07 & $0.92[0.84-0.98]$ & 0.96 \\
\hline & $<56$ & 3 & $0.92[0.88-0.96]$ & & $0.91[0.84-0.99]$ & \\
\hline \multirow[t]{2}{*}{ Sample } & $>50$ & 4 & $0.88[0.82-0.95]$ & 0.15 & $0.93[0.81-0.97]$ & 0.03 \\
\hline & $\leq 50$ & 3 & 0.88 [0.78-0.98] & & $0.85[0.78-0.99]$ & \\
\hline \multirow[t]{2}{*}{ Vendor } & Just GE & 3 & 0.94 [0.90-0.98] & 0.33 & $0.85[0.76-0.94]$ & 0.10 \\
\hline & With Siemens & 3 & 0.82 [0.76-0.89] & & $0.85[0.76-0.94]$ & \\
\hline \multirow[t]{2}{*}{ Cutoff value } & $\geq 3.0$ & 4 & $0.91[0.86-0.95]$ & 0.01 & 0.90 [0.82-0.99] & 0.51 \\
\hline & $<3.0$ & 3 & 0.84 [0.78-0.90] & & 0.87 [0.78-0.97] & \\
\hline \multirow[t]{2}{*}{ Time between FDG administration and scanning } & $\geq 60 \min$ & 3 & 0.94 [0.88-0.99] & 0.72 & 0.89 [0.80-0.99] & 0.43 \\
\hline & $<60 \min$ & 4 & $0.85[0.78-0.91]$ & & $0.88[0.78-0.97]$ & \\
\hline \multirow[t]{2}{*}{ FDG dose } & $\geq 4.0 \mathrm{MBq} / \mathrm{kg}$ & 3 & $0.88[0.78-0.97]$ & 0.15 & $0.89[0.77-1.00]$ & 0.55 \\
\hline & $<4.0 \mathrm{MBq} / \mathrm{kg}$ & 3 & 0.86 [0.79-0.93] & & 0.91 [0.81-1.00] & \\
\hline \multicolumn{7}{|l|}{ ADC } \\
\hline \multirow[t]{2}{*}{ China } & yes & 4 & 0.90 [0.85-0.95] & 0.29 & 0.80 [0.69-0.90] & 0.19 \\
\hline & no & 3 & $0.82[0.72-0.92]$ & & $0.81[0.68-0.94]$ & \\
\hline \multirow[t]{2}{*}{ No. of tumors } & $\geq 100$ & 3 & $0.91[0.88-0.95]$ & 0.02 & $0.78[0.67-0.89]$ & 0.08 \\
\hline & $<100$ & 4 & $0.82[0.75-0.88]$ & & $0.82[0.71-0.93]$ & \\
\hline \multirow[t]{2}{*}{ Max b value } & $1,000 \mathrm{~s} / \mathrm{mm}^{2}$ & 4 & $0.91[0.89-0.94]$ & 0.05 & 0.79 [0.70-0.89] & 0.04 \\
\hline & $800 \mathrm{~s} / \mathrm{mm}^{2}$ & 3 & $0.77[0.70-0.84]$ & & 0.84 [0.68-0.95] & \\
\hline \multirow[t]{2}{*}{ No. of imaging planes } & 3 & 3 & $0.87[0.79-0.96]$ & 0.07 & $0.82[0.71-0.93]$ & 0.29 \\
\hline & 2 & 4 & 0.87 [0.80-0.94] & & 0.79 [0.68-0.90] & \\
\hline \multirow[t]{2}{*}{ Slice thickness } & $6 \mathrm{~mm}$ & 3 & $0.84[0.78-0.95]$ & 0.04 & $0.84[0.75-0.93]$ & 0.16 \\
\hline & $5 \mathrm{~mm}$ & 4 & 0.89 [0.80-0.95] & & $0.76[0.64-0.87]$ & \\
\hline \multirow[t]{2}{*}{ Vendor } & GE & 4 & $0.89[0.84-0.95]$ & 0.22 & $0.78[0.67-0.89]$ & 0.07 \\
\hline & Siemens & 3 & $0.83[0.74-0.93]$ & & $0.83[0.72-0.94]$ & \\
\hline
\end{tabular}

SUVmax, Maximum uptake value; ADC, Apparent diffusion coefficient.

$p<0.05$ indicates that the comparison between groups is statistically significant and is indicated in bold.

indicating that quantitative $\mathrm{ADC}$ value is an useful diagnostic parameter for it.

The present meta-analysis shows heterogeneity in the pooled sensitivity and specificity of studies for SUVmax and ADC. The results of meta regression analysis showed that the sample size of the enrolled studies and the cutoff value of SUVmax were the factors for the heterogeneity between studies using SUVmax to evaluate benign and malignant ovarian or adnexal masses. To be specific, studies with cutoff values greater than or equal to 3.0 have a higher sensitivity to detect malignant lesions, which is not difficult to 
explain due to high metabolism of malignant tumors, thus there will be carbohydrate imaging agent aggregation in the lesions, resulting in higher detection of malignant lesions. Studies with a sample size of less than 50 patients showed lower specificity, which may be related to the fact that a small sample size is more likely to lead to higher false negatives. Moreover, prospective studies have higher specificity and lower sensitivity than retrospective studies, and studies conducted by Siemens has higher sensitivity than that conducted by GE only and has same specificity, but none of them are factors that cause heterogeneity $(p>0.05)$. For the study of ADC, study with larger number of ovarian or adnexal masses $(\geq 100)$ has a higher pooled sensitivity, which is also related to the fact that large sample sizes usually lead to smaller false negative results. The results of meta regression analysis also show that the sensitivity of the larger slice thickness is lower, which is related to the fact that smaller lesions are easily missed when the slice thickness is larger, leading to higher false negatives. Moreover, study with the maximum b value of $1,000 \mathrm{~s} / \mathrm{mm}^{2}$ and performed with GE have higher sensitivity than study with the maximum $b$ value of $1,000 \mathrm{~s} / \mathrm{mm}^{2}$ and performed with Siemens, but they are not factors that affects the pooled sensitivity for ADC. In terms of the pooled specificity, the specificity of the study with the maximum $b$ value of $1,000 \mathrm{~s} / \mathrm{mm}^{2}$ is lower than that of the study with the maximum $b$ value of $800 \mathrm{~s} /$ $\mathrm{mm}^{2}$, since a larger b value improves the contrast of the image while also reducing its signal-to-noise ratio. Therefore, in order to ensure a good contrast-to-noise ratio and signal-to-noise ratio, the normalized $b$ value should be used in future studies and avoid using a too large $b$ value to calculate the ADC (48). Moreover, the results of meta-regression showed that the scanning slice thickness was $6 \mathrm{~mm}$, the image acquisition using three planes of axial, sagittal, and coronal, and the study performed by Siemens showed higher specificity, but there was no statistical difference.

Previous studies have shown that PET/CT and MRI have good diagnostic performance in the identification of benign and malignant ovarian or adnexal masses $(9,47)$. However, the evaluation method of these studies is a qualitative method based on MRI sequence and PET/CT metabolic parameters, which is particularly vulnerable to the subjective or bias of the researcher. The current meta-analysis results provide estimates for the diagnostic performance of quantitative SUVmax and ADC values in predicting benign and malignant ovarian or adnexal tumors, and our study indicates that quantitative both SUVmax and ADC values are useful diagnostic parameters for differentiating malignant and benign ovarian masses.

The main limitation of the current meta-analysis is that the number of eligible studies is relatively limited, while some published related studies did not specify the cutoff value of SUVmax or ADC values for positive explanations, and some studies

\section{REFERENCES}

1. Sung H, Ferlay J, Siegel RL, Laversanne M, Soerjomataram I, Jemal A, et al. Global Cancer Statistics 2020: GLOBOCAN Estimates of Incidence and Mortality Worldwide for 36 Cancers in 185 Countries. CA Cancer J Clin (2021) 71:209-49. doi: 10.3322/caac.21660 cannot obtain TP, FP, FN, and TN values to calculate sensitivity and specificity. Secondly, the heterogeneity in the evaluation of diagnostic accuracy in both SUVmax and ADC studies also limits the quality of this meta-analysis. Finally, the indirect comparison of the diagnostic performance of SUVmax and ADC in differentiating benign and malignant ovarian or adnexal masses is also one of the limitations of the study, so prospective comparative studies are needed in future work. However, the diagnostic performance of quantitative SUVmax and ADC values of the study in differentiating benign and malignant ovarian or adnexal masses provides a reference for clinical practice and avoids the subjective interpretation of results.

In conclusion, quantitative SUVmax and ADC values showed to have high diagnostic performance in differentiating benign and malignant ovarian or adnexal masses, and the diagnostic accuracy of quantitative SUVmax is higher, both of which can be used as useful diagnostic parameters for the diagnosis of ovarian cancer. Large-scale sample size and high-quality trials to evaluate and verify the clinical value of quantitative SUVmax and ADC values in the diagnosis of ovarian cancer are needed in future work.

\section{DATA AVAILABILITY STATEMENT}

The original contributions presented in the study are included in the article/Supplementary Material. Further inquiries can be directed to the corresponding authors.

\section{AUTHOR CONTRIBUTIONS}

$\mathrm{XH}$ and $\mathrm{ZL}$ conceptualized the study, revised the manuscript and supervised the study. CZ and GW conceptualized the study, drafted the manuscript and made the figures. $\mathrm{XH}$, JC and PW collected the literature and revised the manuscript. All authors contributed to the article and approved the submitted version.

\section{FUNDING}

This study was funded by the National Natural Science Foundation of the Peoples Republic of China, NSFC (grant numbers: 81571712), and Qiankehe platform talents[2017] (grant numbers: 5733-035). None declared from all other authors.

\section{SUPPLEMENTARY MATERIAL}

The Supplementary Material for this article can be found online at: https://www.frontiersin.org/articles/10.3389/fonc.2022.840433/ full\#supplementary-material

2. Conic I, Dimov I, Tasic-Dimov D, Djordjevic B, Stefanovic V. Ovarian Epithelial Cancer Stem Cells. Sci World J (2011) 11:1243-69. doi: 10.1100/tsw.2011.112

3. Terry KL, Schock H, Fortner RT, Hüsing A, Fichorova RN, Yamamoto HS, et al. A Prospective Evaluation of Early Detection Biomarkers for Ovarian Cancer in the European EPIC Cohort. Clin Cancer Res (2016) 22:4664-75. doi: 10.1158/1078-0432.CCR-16-0316 
4. Kipps E, Tan DS, Kaye SB. Meeting the Challenge of Ascites in Ovarian Cancer: New Avenues for Therapy and Research. Nat Rev Cancer (2013) 13:273-82. doi: 10.1038/nrc3432

5. Zhang M, Zhang Y, Fu J, Zhang L. Serum CA125 Levels Are Decreased in Rectal Cancer But Increased in Fibrosis-Associated Diseases and in Most Types of Cancers. Prog Mol Biol Transl Sci (2019) 162:241-52. doi: 10.1016/ bs.pmbts.2018.12.012

6. Biggs WS, Marks ST. Diagnosis and Management of Adnexal Masses. Am Fam Physician (2016) 93:676-81.

7. Thomassin-Naggara I, Aubert E, Rockall A, Jalaguier-Coudray A, Rouzier R, Daraï E, et al. Adnexal Masses: Development and Preliminary Validation of an MR Imaging Scoring System. Radiol (2013) 267:432-43. doi: 10.1148/ radiol.13121161

8. Addley H, Moyle P, Freeman S. Diffusion-Weighted Imaging in Gynaecological Malignancy. Clin Radiol (2017) 72:981-90. doi: 10.1016/ j.crad.2017.07.014

9. Hu X, Li D, Liang Z, Liao Y, Yang L, Wang R, et al. Indirect Comparison of the Diagnostic Performance of ${ }^{18} \mathrm{~F}$-FDG PET/CT and MRI in Differentiating Benign and Malignant Ovarian or Adnexal Tumors: A Systematic Review and MetaAnalysis. BMC Cancer (2021) 21:1080. doi: 10.1186/s12885-021-08815-3

10. Moher D, Liberati A, Tetzlaff J, Altman DG. PRISMA Group. Preferred Reporting Items for Systematic Reviews and Meta-Analyses: The PRISMA Statement. PloS Med (2009) 6:e1000097.

11. Whiting PF, Rutjes AW, Westwood ME, Mallett S, Deeks JJ, Reitsma JB, et al. QUADAS-2: A Revised Tool for the Quality Assessment of Diagnostic Accuracy Studies. Ann Intern Med (2011) 155:529-36. doi: 10.7326/00034819-155-8-201110180-00009

12. Higgins JP, Thompson SG, Deeks JJ, Altman DG. Measuring Inconsistency in Meta-Analyses. BMJ (2003) 327:557-60. doi: 10.1136/bmj.327.7414.557

13. Deeks JJ, Macaskill P, Irwig L. The Performance of Tests of Publication Bias and Other Sample Size Effects in Systematic Reviews of Diagnostic Test Accuracy was Assessed. J Clin Epidemiol (2005) 58:882-93. doi: 10.1016/ j.jclinepi.2005.01.016

14. Castellucci P, Perrone AM, Picchio M, Ghi T, Farsad M, Nanni C, et al. Diagnostic Accuracy of ${ }^{18}$ F-FDG PET/CT in Characterizing Ovarian Lesions and Staging Ovarian Cancer: Correlation With Transvaginal Ultrasonography, Computed Tomography, and Histology. Nucl Med Commun (2007) 28:589-95. doi: 10.1097/MNM.0b013e3281afa256

15. Yamamoto Y, Oguri H, Yamada R, Maeda N, Kohsaki S, Fukaya T. Preoperative Evaluation of Pelvic Masses With Combined ${ }^{18}$ Ffluorodeoxyglucose Positron Emission Tomography and Computed Tomography. Int J Gynaecol Obstet (2008) 102:124-7. doi: 10.1016/j.ijgo.2008.02.019

16. Kitajima K, Suzuki K, Senda M, Kita M, Nakamoto Y, Onishi Y, et al. FDGPET/ CT for Diagnosis of Primary Ovarian Cancer. Nucl Med Commun (2011) 32:549-53. doi: 10.1097/MNM.0b013e328345b339

17. Zytoon AA, Murakami K, Eid H, El-Gammal M. High Impact of FDG-PET/ CT in Diagnostic Strategies for Ovarian Cancer. Acta Radiol (2013) 54:340-8. doi: 10.1258/ar.2012.120632

18. Tanizaki Y, Kobayashi A, Shiro M, Ota N, Takano R, Mabuchi Y, et al. Diagnostic Value of Preoperative SUVmax on FDG-PET/CT for the Detection of Ovarian Cancer. Int J Gynecol Cancer (2014) 24:454-60. doi: 10.1097/ IGC.0000000000000074

19. Lee JW, Lee JH, Cho A, Yun M, Lee JD, Kim YT, et al. The Performance of Contrast-Enhanced FDG PET/CT for the Differential Diagnosis of Unexpected Ovarian Mass Lesions in Patients With Nongynecologic Cancer. Clin Nucl Med (2015) 40:97-102. doi: 10.1097/RLU.0000000000000667

20. Takagi H, Sakamoto J, Osaka Y, Shibata T, Fujita S, Sasagawa T. Utility of ${ }^{18}$ Ffluorodeoxyglucose-Positron Emission Tomography in the Differential Diagnosis of Benign and Malignant Gynaecological Tumours. J Med Imaging Radiat Oncol (2018) 62:471-9. doi: 10.1111/1754-9485.12707

21. Li W, Chu C, Cui Y, Zhang P, Zhu M. Diffusion-Weighted MRI: A Useful Technique to Discriminate Benign Versus Malignant Ovarian Surface Epithelial Tumors With Solid and Cystic Components. Abdom Imaging (2012) 37:897-903. doi: 10.1007/s00261-011-9814-X

22. Zhang P, Cui Y, Li W, Ren G, Chu C, Wu X. Diagnostic Accuracy of Diffusionweighted Imaging With Conventional MR Imaging for Differentiating Complex Solid and Cystic Ovarian Tumors at 1.5T. World J Surg Oncol (2012) 10:237. doi: 10.1186/1477-7819-10-237
23. Fan X, Zhang H, Meng S, Zhang J, Zhang C. Role of Diffusion-Weighted Magnetic Resonance Imaging in Differentiating Malignancies From Benign Ovarian Tumors. Int J Clin Exp Med (2015) 8:19928-37.

24. Zhang H, Mao Y, Chen X, Wu G, Liu X, Zhang P, et al. Magnetic Resonance Imaging Radiomics in Categorizing Ovarian Masses and Predicting Clinical Outcome: A Preliminary Study. Eur Radiol (2019) 29:3358-71. doi: 10.1007/ s00330-019-06124-9

25. Türkoğlu S, Kayan M. Differentiation Between Benign and Malignant Ovarian Masses Using Multiparametric MRI. Diagn Interv Imaging (2020) 101:147-55. doi: 10.1016/j.diii.2020.01.006

26. Mansour S, Wessam R, Raafat M. Diffusion-Weighted Magnetic Resonance Imaging in the Assessment of Ovarian Masses With Suspicious Features: Strengths and Challenges. Egyp J Radiol Nucl Med (2015) 46:1279-89. doi: 10.1016/j.ejrnm.2015.06.023

27. Takeuchi M, Matsuzaki K, Nishitani H. Diffusionweighted Magnetic Resonance Imaging of Ovarian Tumors: Differentiation of Benign and Malignant Solid Components of Ovarian Masses. J Comput Assist Tomogr (2010) 34:173-6. doi: 10.1097/RCT.0b013e3181c2f0a2

28. Lee MI, Jung YJ, Kim DI, Lee S, Jung CS, Kang SK, et al. Prognostic Value of SUVmax in Breast Cancer and Comparative Analyses of Molecular Subtypes: A Systematic Review and Meta-Analysis. Med (Baltimore) (2021) 100:e26745. doi: 10.1097/MD.0000000000026745

29. Han L, Wang Q, Zhao L, Feng X, Wang Y, Zou Y, et al. A Systematic Review and Meta-Analysis of the Prognostic Impact of Pretreatment Fluorodeoxyglucose Positron Emission Tomography/Computed Tomography Parameters in Patients With Locally Advanced Cervical Cancer Treated With Concomitant Chemoradiotherapy. Diagnostics (Basel) (2021) 11:1258. doi: 10.3390/ diagnostics 11071258

30. Zhang Q, Xi Y, Li D, Yuan Z, Dong J. The Utility of ${ }^{18}$ F-FDG PET and PET/ CT in the Diagnosis and Staging of Chondrosarcoma: A Meta-Analysis. J Orthop Surg Res (2020) 15:229. doi: 10.1186/s13018-020-01748-w

31. Xuan D, Wen W, Tian S, Piao M, Xu D, Liu L. Prognostic Value of Maximum Standard Uptake Value, Metabolic Tumor Volume, and Total Lesion Glycolysis of 18F-FDG PET/CT in Patients With Renal Carcinoma: A Protocol for Systematic Review and Meta Analysis. Med (Baltimore) (2020) 99:e19988. doi: 10.1097/MD.0000000000019988

32. Wen W, Xuan D, Hu Y, Li X, Liu L, Xu D. Prognostic Value of Maximum Standard Uptake Value, Metabolic Tumor Volume, and Total Lesion Glycolysis of Positron Emission Tomography/Computed Tomography in Patients With Breast Cancer: A Systematic Review and Meta-Analysis. PloS One (2019) 14:e0225959. doi: 10.1371/journal.pone.0225959

33. Wang H, Shen G, Jiang C, Li L, Cui F, Tian R. Prognostic Value of Baseline, Interim and End-of-Treatment ${ }^{18} \mathrm{~F}$-FDG PET/CT Parameters in Extranodal Natural Killer/T-Cell Lymphoma: A Meta-Analysis. PloS One (2018) 13: e0194435. doi: 10.1371/journal.pone.0194435

34. Sarker A, Im HJ, Cheon GJ, Chung HH, Kang KW, Chung JK, et al. Prognostic Implications of the SUVmax of Primary Tumors and Metastatic Lymph Node Measured by ${ }^{18}$ F-FDG PET in Patients With Uterine Cervical Cancer: A Meta-Analysis. Clin Nucl Med (2016) 41:34-40. doi: 10.1097/RLU.0000000 000001049

35. Meyer HJ, Gundermann P, Surov A. Associations Between FDG-PET and Ki 67-Index in Head and Neck Cancer: A Meta-Analysis. Med (Baltimore) (2019) 98:e17472. doi: 10.1097/MD.0000000000017472

36. Shen G, Ma H, Pang F, Ren P, Kuang A. Correlations of ${ }^{18}$ F-FDG and ${ }^{18}$ F-FLT Uptake on PET With Ki-67 Expression in Patients With Lung Cancer: A MetaAnalysis. Acta Radiol (2018) 59:188-95. doi: 10.1177/0284185117706609

37. Herneth AM, Philipp MO, Naude J, Funovics M, Beichel RR, Bammer R, et al. Vertebral Metastases: Assessment With Apparent Diffusion Coefficient. Radiol (2002) 225:889-94. doi: 10.1148/radiol.2253011707

38. Plank C, Koller A, Mueller-Mang C, Bammer R, Thurnher MM. Diffusionweighted MR Imaging (DWI) in the Evaluation of Epidural Spinal Lesions. Neuroradiol (2007) 49:977-85. doi: 10.1007/s00234-007-0275-4

39. Dury RJ, Lourdusamy A, Macarthur DC, Peet AC, Auer DP, Grundy RG, et al. Meta-Analysis of Apparent Diffusion Coefficient in Pediatric Medulloblastoma, Ependymoma, and Pilocytic Astrocytoma. J Magn Reson Imaging (2021) 2021:34842328. doi: 10.1002/jmri.28007

40. Meyer HJ, Wienke A, Surov A. Discrimination Between Malignant and Benign Thyroid Tumors by Diffusion-Weighted Imaging - A Systematic 
Review and Meta Analysis. Magn Reson Imaging (2021) 84:41-57. doi: 10.1016/j.mri.2021.09.006

41. Zhu M, Zhang C, Yan J, Sun J, Zhao X, Zhang L, et al. Accuracy of Quantitative Diffusion-Weighted Imaging for Differentiating Benign and Malignant Pancreatic Lesions: A Systematic Review and Meta-Analysis. Eur Radiol (2021) 31:7746-59. doi: 10.1007/s00330-021-07880-3

42. Virarkar M, Diab R, Palmquist S, Bassett R Jr, Bhosale P. Diagnostic Performance of MRI to Differentiate Uterine Leiomyosarcoma From Benign Leiomyoma: A Meta-Analysis. J Belg Soc Radiol (2020) 104:69. doi: 10.5334/jbsr.2275

43. Drewes R, Heinze C, Pech M, Powerski M, Woidacki K, Wienke A, et al. Apparent Diffusion Coefficient can Predict Therapy Response of Hepatocellular Carcinoma to Transcatheter Arterial Chemoembolization. Dig Dis (2021) 2021:34749359. doi: 10.1159/000520716

44. Lee MK, Choi Y, Jung SL. Diffusion-Weighted MRI for Predicting Treatment Response in Patients With Nasopharyngeal Carcinoma: A Systematic Review and Meta-Analysis. Sci Rep (2021) 11:18986. doi: 10.1038/s41598-021-98508-5

45. Drewes R, Pech M, Powerski M, Omari J, Heinze C, Damm R, et al. Apparent Diffusion Coefficient Can Predict Response to Chemotherapy of Liver Metastases in Colorectal Cancer. Acad Radiol (2021) 28(Suppl 1):S73-80. doi: 10.1016/j.acra.2020.09.006

46. Thomassin-Naggara I, Daraï E, Cuenod CA, Fournier L, Toussaint I, Marsault $\mathrm{C}$, et al. Contribution of Diffusion-Weighted MR Imaging for Predicting Benignity of Complex Adnexal Masses. Eur Radiol (2009) 19:1544-52. doi: 10.1007/s00330-009-1299-4

47. Kim HJ, Lee SY, Shin YR, Park CS, Kim K. The Value of DiffusionWeighted Imaging in the Differential Diagnosis of Ovarian Lesions: A
Meta-Analysis. PloS One (2016) 11:e0149465. doi: 10.1371/journal.pone. 0149465

48. Barral M, Taouli B, Guiu B, Koh DM, Luciani A, Manfredi R, et al. DiffusionWeighted MR Imaging of the Pancreas: Current Status and Recommendations. Radiology (2015) 274:45-63. doi: 10.1148/radiol.14130778

Conflict of Interest: Author GW was employed by Jiangsu Yuanben Biotechnology Co., Ltd.

The remaining authors declare that the research was conducted in the absence of any commercial or financial relationships that could be construed as a potential conflict of interest.

Publisher's Note: All claims expressed in this article are solely those of the authors and do not necessarily represent those of their affiliated organizations, or those of the publisher, the editors and the reviewers. Any product that may be evaluated in this article, or claim that may be made by its manufacturer, is not guaranteed or endorsed by the publisher.

Copyright (c) $2022 \mathrm{Hu}$, Liang, Zhang, Wang, Cai and Wang. This is an open-access article distributed under the terms of the Creative Commons Attribution License (CC BY). The use, distribution or reproduction in other forums is permitted, provided the original author(s) and the copyright owner(s) are credited and that the original publication in this journal is cited, in accordance with accepted academic practice. No use, distribution or reproduction is permitted which does not comply with these terms. 